\title{
FETAL HEART RATE AND FETAL HEART RATE VARIABILITY IN LIPIZZANER BROODMARES
}

\author{
Boglárka BASKA-VINCZE ${ }^{1,3^{*}}$, Ferenc BASKA ${ }^{2}$ and Ottó SZENCI ${ }^{3}$ \\ ${ }^{1}$ Equine Department and Clinic, Szent István University, Dóra major, H-2225 Üllö, \\ Hungary; ${ }^{2}$ Department of Pathology, Szent István University, Budapest, Hungary; \\ ${ }^{3}$ Hungarian Academy of Sciences - Large Animal Clinical Research Group, Üllö, Hungary
}

(Received 2 March 2014; accepted 29 October 2014)

\begin{abstract}
Monitoring fetal heart rate (FHR) and fetal heart rate variability (FHRV) helps to understand and evaluate normal and pathological conditions in the foal. The aim of this study was to establish normal heart rate reference values for the ongoing equine pregnancy and to perform a heart rate variability (HRV) timedomain analysis in Lipizzaner mares. Seventeen middle- and late-term (days 121333) pregnant Lipizzaner mares were examined using fetomaternal electrocardiography $(E C G)$. The mean FHR $(P=0.004)$ and the standard deviation of FHR $(\mathrm{P}=0.012)$ significantly decreased during the pregnancy. $\mathrm{FHR} \pm \mathrm{SD}$ values decreased from $115 \pm 35$ to $79 \pm 9 \mathrm{bpm}$ between months 5 and 11 . Our data showed that HRV in the foal decreased as the pregnancy progressed, which is in contrast with the findings of earlier equine studies. The standard deviation of normalnormal intervals (SDNN) was higher $(70 \pm 25$ to $166 \pm 108 \mathrm{msec})$ than described previously. The root mean square of successive differences (RMSSD) decreased from $105 \pm 69$ to $77 \pm 37 \mathrm{msec}$ between the 5th and 11th month of gestation. Using telemetric ECG equipment, we could detect equine fetal heartbeat on day 121 for the first time. In addition, the large differences observed in the HR values of four mare-fetus pairs in four consecutive months support the assumption that there might be 'high-HR' and 'low-HR' fetuses in horses. It can be concluded that the analysis of FHR and FHRV is a promising tool for the assessment of fetal well-being but the applicability of these parameters in the clinical setting and in studs requires further investigation.
\end{abstract} analysis

Key words: Fetal ECG, equine, well-being, heart rate, heart rate variability,

Transrectal and transabdominal ultrasonography is the most commonly used method for the assessment of fetal well-being in the second half of gestation in horses (Reef et al., 1995, 1996; Bucca et al., 2005; Baska-Vincze et al., 2013, 2014). Depending on the duration of the examination, ultrasonography provides the veterinarian with useful data about the fetus, makes it possible to measure

*Corresponding author; E-mail: Vincze.Boglarka@aotk.szie.hu; Phone: 0036 (20) 259-4909; Fax: 0036 (29) 521-303 
specific dimensions and values of the fetal body and to study the environment surrounding it (fetal membranes, fetal fluids, uterus). One of the most important elements of the examination is the measurement of fetal heart rate (FHR), which can be done using M-mode echography. However, even this method has some limitations: it may be difficult to carry out because of the maternal and fetal movements (Baska-Vincze et al., 2014). A successful measurement shows the heart rate of the fetus at a given moment, which is markedly influenced by fetal activity (Reef et al., 1995, 1996; Palmer, 2000; Baska-Vincze et al., 2014). Measurement and evaluation of FHR have outstanding importance as the fetus responds to the decrease of oxygen supply in a manner completely different from the response of adult animals. In adult humans and domestic animals, tissue hypoxia triggers a dramatic increase in heart rate and respiratory rate in order to supply the organs with sufficient oxygen. For the fetus, however, it is not possible to increase the volume of blood and oxygen delivered to the uterus. First the fetal heart rate decreases, in order to reduce the work of the heart and the oxygen demand of the myocardium. Simultaneously, vasoconstriction occurs in the organs to make sure that the brain, the heart and the adrenal glands get sufficient oxygen. All this is possible because the fetus can tolerate a short-term hypoxaemia without sustaining any damage. In fetuses with an intact central nervous system the placental perfusion slows down, increasing the amount of oxygen reaching the fetal blood circulation. If decreased oxygen supply persists, the fetus is forced to make further adaptations. Certain processes (such as growth, anabolic processes, movement) are not necessary for short-term survival. Through a mechanism that is yet unclear, the fetus can temporarily arrest these processes for the duration of hypoxaemia. The fetus responds to the stress caused by hypoxia with bradycardia and with the absence of fetal respiratory and other movements. If the insufficiency of oxygen supply is aggravated further or persists, compensatory mechanisms aimed at maintaining the minimum perfusion will be triggered, resulting in tachycardia which is not accompanied by movement activity. When the myocardium becomes exhausted, bradycardia develops again, immediately prior to death (Long and Henry, 1998; Adamson, 1999; Palmer, 2000).

In human obstetrics, the heart rate of the fetus and the associated fetal movements are studied by the so-called cardiotocography after the 30th week of pregnancy. In humans, the variability of fetal heart rate increases markedly in the third trimester of pregnancy. This can probably be attributed to the maturation of the vegetative nervous system which influences cardiac function (Wheeler et al., 1979; van Leeuwen et al., 1999).

In horses, electrocardiographic examinations have been performed for almost 100 years (Nörr, 1921), and since the 1970s ECG has been routinely used on adult horses under clinical conditions. However, fetomaternal ECG examination, which records signals from the fetus in addition to the mare, became accessible to clinicians only in the late 2000 s, with the advent of the new, so-called 
telemetric ECG systems. Nagel et al. have reported data on the heart rate and heart rate variability of equine fetuses since 2010 . Their original objective was to determine physiological ranges of the heart rate (beats per minute) of equine fetuses in different months of gestation. They could successfully measure the fetal heart rate from day 173 of gestation onwards. They collected data about warmblood $(n=7-15)$ and Shetland $(n=7)$ mares and their fetuses, but reported physiological ranges only for days $170-240(n=13)$ and day $320(n=3-7)$ of gestation, as well as for day -1 before parturition $(n=3-7)$ (Nagel et al., 2010, 2011). From their measurements they concluded that FHR continuously decreases while HRV increases with the advancement of gestation (Nagel et al., 2010, 2011).

Correct interpretation of the above findings requires an overview of the definitions of HRV and FHRV. HRV numerically expresses and describes the variability in heart rate between two consecutive beats, caused by the influence of the neuroendocrine system. The heart rate is not constant even in a healthy animal or at rest, as the distances between two consecutive $\mathrm{R}$ waves (the RR intervals) are not the same. The reason for this is that the heart is under the influence of the neuroendocrine system and the vegetative nervous system. The function of this highly complex, multi-step system is to maintain the physiological arterial pressure. Thus, HRV numerically expresses the neurohormonal effect exerted on the heart rate. In general, it can be stated that the higher the HRV, the healthier the heart (Bowen, 2010).

Data have been published about HRV in pregnant cows as well (Kovács et al., 2012; Kovács et al., 2013). The measurement of HRV was reported to be an accepted method for assessing the level of stress, as during stress the activity of the sympathetic nervous system increases and certain HRV values change (Kovács et al., 2012; Kovács et al., 2013). According to other authors, however, the HRV values do not change significantly either with age or during pregnancy (Minero et al., 2001; Mohr et al., 2002).

HRV analysis requires a good-quality ECG. For this, the animals must be kept in a well-known and familiar environment and the stress factors potentially leading to artefacts or non-valid results must be eliminated. Opinions vary as to the required duration of the ECG recording: some authors claim that 24-hour or 4-hour recordings are needed (Bowen, 2010), but since 2007 (von Borell et al., 2007 ) the analysis of time periods as short as 5 minutes has been considered acceptable in both human and veterinary medicine during different HRV studies. When recording the ECG, it is advisable to use electronic filters (so-called artefact filters), which are built-in accessories of the majority of ECG machines (Bowen, 2010).

ECG recordings can be analysed for HRV in different ways, e.g. according to the time domain, the frequency domain and in a non-linear manner. Timedomain analysis is the most common, as it allows the analysis of recordings of both shorter and longer duration, and the result is relatively independent of arte- 
facts. Clinically, this analysis has proved to be the most useful in horses. The two most important parameters belonging here are the standard deviation of normalnormal intervals (SDNN) and the root mean square of successive differences (RMSSD). The former depends on the regulatory effect of the sympathetic and the parasympathetic nervous system, while the latter reflects the long-term variability of heart function and its value primarily depends on the parasympathetic tone (Bowen, 2010; Kovács et al., 2012, 2013). Frequency-domain analysis aims at discriminating the effects of different elements of the neuroendocrine system. It studies how many per cent of the signals falls within a specific frequency domain. Within this system, three factors influence cardiac function: the reninangiotensin-aldosterone (RAAS) system that acts on the heart rate for a longer period of time (seconds or minutes), the sympathetic nervous system (seconds) and the parasympathetic nervous system (during the RR interval). This method of analysis (also called spectral analysis) differentiates three frequency domains: a high-frequency, a low-frequency and a very low frequency range. It should be mentioned that, before running the analysis, the frequency domains established for horses must be set in the computer programme, as these differ from those used in human medicine (von Borell et al., 2007; Bowen, 2010). Within the frequency-domain analysis, the so-called LF/HF ratio, which is an indicator of sympathetic-parasympathetic balance and sympathetic activity, is very important. If the animal is exposed to stress of shorter or longer duration, the HF part decreases and thus the LF/HF ratio increases (Kovács et al., 2012, 2013).

It is known from previous studies that the heart rate of the human fetus decreases from month to month with the advancement of pregnancy (Wheeler et al., 1979; van Leeuwen et al., 1999). Nagel et al. (2010, 2011) have found that the same occurs in the equine fetus, with an increase of HRV during gestation. Time-domain analysis has demonstrated a significant increase in the mean RR intervals and SDNN with the progression of gestation (Nagel et al., 2010, 2011). According to reports in the international literature, stress (e.g. transport), pain and fetal distress result in a decrease of HRV (Bowen, 2010; Nagel et al., 2010).

Studies on human fetuses have revealed that certain circumstances (condition of the mother, stressful situations) play a role in the development of fetal activity and FHR. In the first trimester of pregnancy, the intra-individual FHR variation is low; however, a recently published study noted differences between the FHR and HRV values of individual fetuses in the third trimester of pregnancy. A total of 11 fetuses were examined to determine whether there were differences between the FHR values of individual fetuses. The analysis of 5-minute ECG recordings of fetuses demonstrated that, in the course of pregnancy, the intra-individual variation of FHR was lower than the difference of FHR from that of other fetuses of the same age and health status (van Leeuwen et al., 2013). It has also been established that HRV is influenced by HR, as a higher HR was associated with a lower HRV (van Leeuwen et al., 2013). 
The objective of this study was to collect data on the heart rate and heart rate variability of the fetuses of Lipizzaner broodmares during a normal pregnancy and to determine whether there are individual differences in the mean heart rate of equine fetuses during the final stage of gestation.

\section{Materials and methods}

Seventeen middle- and late-term (days 121-333) pregnant Lipizzaner mares were examined using fetomaternal electrocardiography. Gestational age was determined as the number of days after the last artificial insemination of each mare. All the mares (aged 6-18 years) had been considered clinically healthy (symptomless, with normal heart rate, respiratory rate and rectal temperature) with normal pregnancies. One to five recordings per mare were made. The examinations took place in the examination room of a Hungarian stud farm in the morning hours between 9 a.m. and 12 a.m. All of the mares were familiar with the place, and were kept in a stock during the examination.

For the ECG recordings, a Televet 100 Holter ECG equipment (Engel Engineering Services $\mathrm{GmbH}$, Heusenstamm, Germany) was used as described by Nagel et al. (2010). At least 15-minute-long recordings were made during each examination. As proposed by von Borell et al. (2007), five-minute-long ECG recordings were cut and saved for HRV analysis using the Kubios HRV software (Biosignal Analysis and Medical Imaging Group, Kuopio, Finland). For the 5min ECG analysis, the continuous fetal sign was the criterion. A sample ECG recording is shown in Fig. 1. To avoid artefacts, an artefact filter was used, and the data had been detrended before the analysis. In this study, time-domain analysis was performed and, thus, the mean FHR, the standard deviation of FHR, the mean RR interval, the standard deviation of normal-normal intervals (SDNN) and the root mean square of successive differences (RMSSD) were evaluated. Statistical analysis was performed using Excel 2007 (Microsoft Corporation, Washington, USA) and R (R Core Team, Vienna, Austria) software (R Core Team, 2013). To evaluate the normal distribution of the values, the Shapiro-Wilk test was used. In case of normalcy, Pearson's correlation analysis was used, in other cases, the authors used Spearman's rank correlation analysis. The variables for time-domain analysis were compared to gestational age in days. A significance level of $\mathrm{P}<0.05$ was selected for all analyses performed on the data.

\section{Results}

Seventeen non-sedated mid- and late-pregnant Lipizzaner mares (gestational days 121-333) were examined monthly from November 2013, and a total of 37 ECG recordings (1-5 recordings per mare) were made and analysed. 


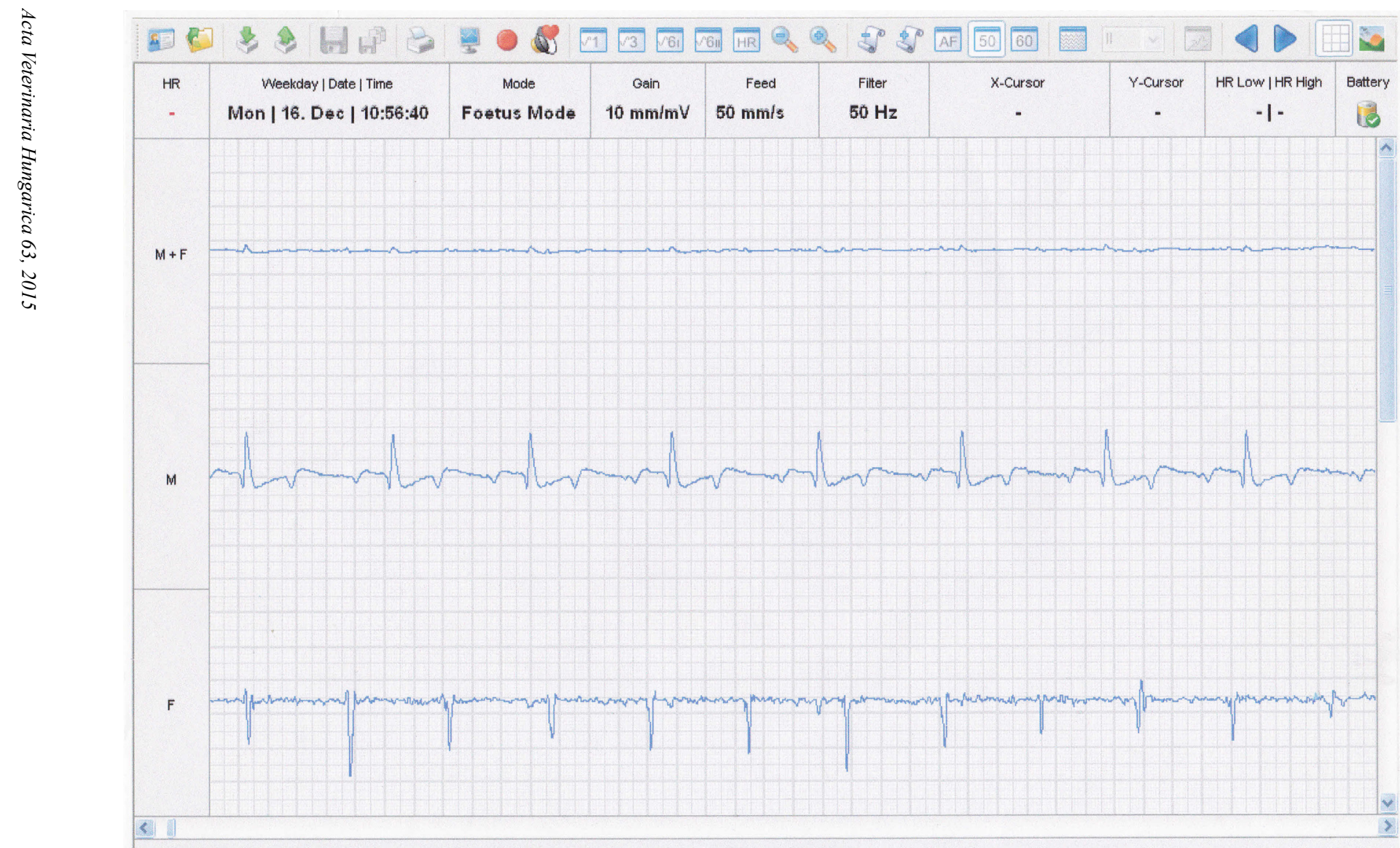

267 \#0003. Birthdate 01. Jan 2000 | ECG \#00009. 16. Dect 2013. Starttime 10:56:25

Fig. 1. Fetomaternal ECG recording (Televet software, Engel Engineering Ltd., Germany) 
The signal of the fetal heartbeat could be detected and recorded from day 121 (the fifth month) of equine pregnancy.

The FHR $(\mathrm{FHR} \pm \mathrm{SD}$, bpm) and HRV (mean $\pm \mathrm{SD}$, ms) values in the different gestational months are presented in Table 1.

\section{Table 1}

Heart rate and heart rate variability values of the equine fetus in different months of pregnancy

\begin{tabular}{lccccc}
\hline $\begin{array}{l}\text { HR and HRV } \\
\text { values }\end{array}$ & $\begin{array}{c}\text { Months 5-7 } \\
(\mathrm{n}=7)\end{array}$ & $\begin{array}{c}\text { Month 8 } \\
(\mathrm{n}=6)\end{array}$ & $\begin{array}{c}\text { Month 9 } \\
(\mathrm{n}=6)\end{array}$ & $\begin{array}{c}\text { Month 10 } \\
(\mathrm{n}=9)\end{array}$ & $\begin{array}{c}\text { Month 11 } \\
(\mathrm{n}=9)\end{array}$ \\
\hline Mean FHR, bpm & $115 \pm 35$ & $91 \pm 10$ & $92 \pm 9$ & $85 \pm 9$ & $79 \pm 9$ \\
SDNN, msec & $166 \pm 108$ & $83 \pm 39$ & $70 \pm 25$ & $88 \pm 65$ & $121 \pm 75$ \\
RMSSD, msec & $105 \pm 69$ & $28 \pm 11$ & $30 \pm 18$ & $40 \pm 35$ & $77 \pm 37$ \\
Mean RR, msec & $698 \pm 119$ & $754 \pm 125$ & $680 \pm 70$ & $740 \pm 68$ & $785 \pm 101$ \\
\hline
\end{tabular}

$\mathrm{HR}=$ heart rate $\mathrm{HRV}=$ heart rate variability $; \mathrm{FHR}=$ fetal heart rate; $\mathrm{SDNN}=$ standard deviation of normal-normal intervals; RMSSD = root mean square of successive differences; $R R=R$ wave to $\mathrm{R}$ wave interval

The mean FHR $(\mathrm{P}=0.004)$ and the standard deviation of FHR $(\mathrm{P}=0.012)$ significantly decreased during the pregnancy; the FHR \pm SD values decreased from $115 \pm 35$ to $79 \pm 9 \mathrm{bpm}$ during the ongoing pregnancy. This decreasing tendency can be seen in Fig. 2. The RR intervals compared to the gestational days tended to increase during pregnancy, but this increase was not significant. SDNN and RMSSD tended to decrease but their decrease did not reach the level of significance.

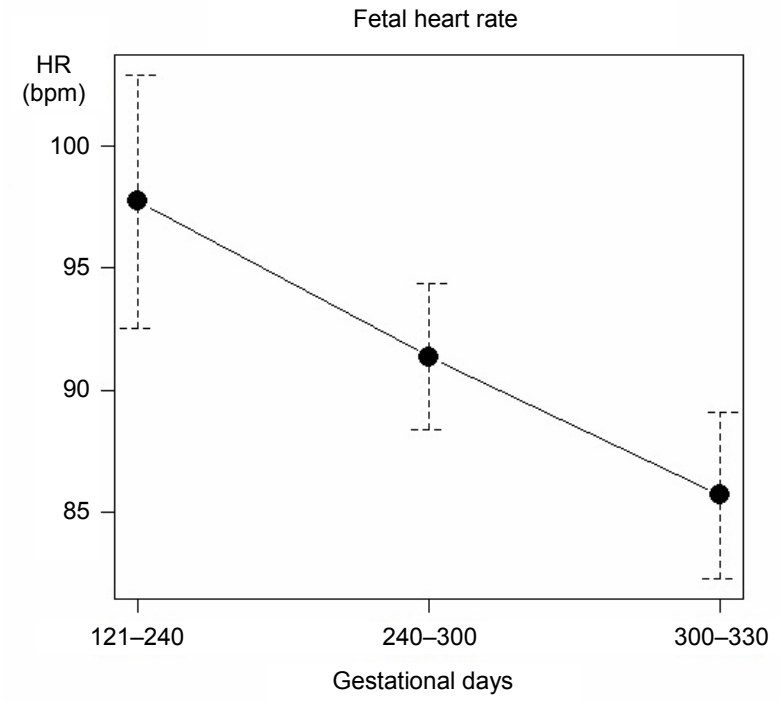

Fig. 2. A plot of means showing decreasing fetal heart rates during gestation (days 121-330) 
In case of four fetuses, four recordings per mare could be made in the same consecutive months $(8,9,10$ and 11) of the last trimester. In these fetuses, the change of FHR values over time could be compared within the same individual. A plot of mean FHR and SDFHR is presented in Fig. 3.

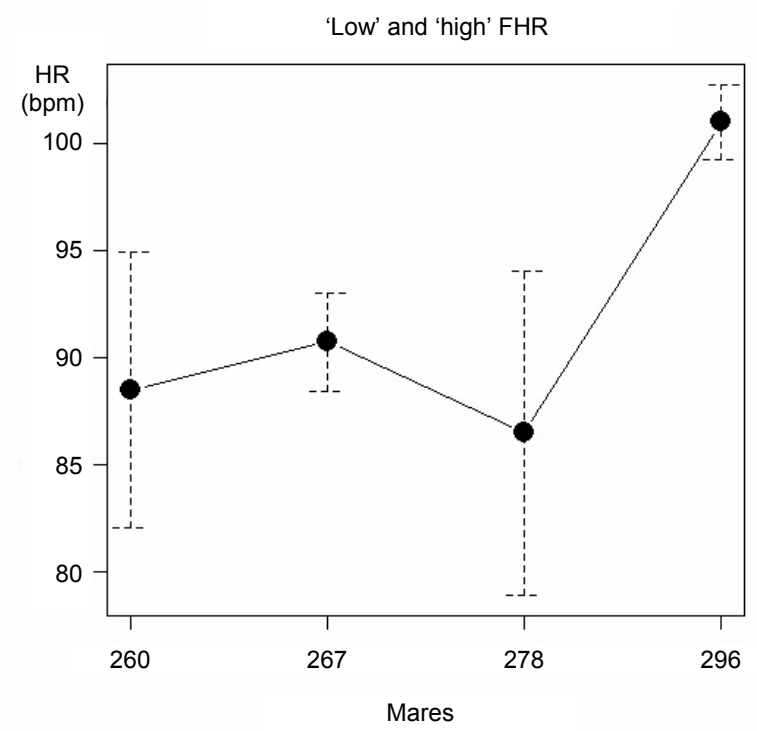

Fig. 3. A plot of means showing fetal heart rates recorded in months $8-11$ of gestation in four pregnant mares (no. 260, 267, 278 and 296) with single fetuses

\section{Discussion}

On the basis of our findings, we can conclude that the telemetric ECG Holter equipment can easily be used under farm conditions, as suggested by Nagel et al. (2010). This non-invasive technique provides useful data about the well-being of the equine fetus and renders it possible to make either short (e.g. 15-min) and long continuous (e.g. 24-hour) recordings on pregnant mares. Originally it was recommended to use long (e.g. 4- or 24-hour) continuous recordings on horses, but in our study it was not possible to restrain or follow the mares at rest for such a long time. These mares are kept in runner stables and on pasture during the day, and a maximum time of 15-20 minutes is adequate for them in the examination building because they are familiar with the place but a longer time spent there would be stressful for these animals. If we had used a longer examination period, the results would have been false because of the effect of elevated blood cortisol levels on HR and HRV. During the examinations the horses generally remained calm and the recording was stopped if they started to behave restlessly. Theoretically, the long recording time could be more representative in 
the case of HRV analysis, but we strongly recommend to use a 5-minute recording because of the transformation process of the data from ECG to an RR text file which is accepted by the HRV analysis programme (Kubios HRV software). The adaptation of a long ECG recording to a text file would be time consuming and impractical. For work organisation reasons, evaluations were made in the morning hours (between 9 and 12 a.m.). It has been described that this is the period when plasma cortisol reaches its peak in the adult horse (Cordero et al., 2012). As handling could theoretically cause an increased sympathetic tone in the mares (even if they were familiar with the environment), this could influence their cardiovascular status which might have an effect on the fetuses at the time of the recording. Hormones of relatively small molecule size, such as cortisol, can cross the placenta (Senger, 2012); however, to the best of the authors' knowledge their theoretical effect on the fetus and fetal heart rate has not been elucidated yet. In the authors' opinion, in this study FHR was not markedly influenced by the suspected elevation of maternal cortisol level, because the HR values were rather similar to those reported by Nagel et al. (2010). This is supported by the fact that in the first Nagel study, recordings were made at 6 a.m. and 4 p.m., and the authors did not find any difference in HR and HRV values in those two time periods during the day (Nagel et al., 2010).

The aim of this work was to obtain data on FHR and FHRV during the different months of equine gestation in Lipizzaner mares (Table 1), as before this study only data relating to longer periods of time had been available. Nagel et al. (2010) divided their results into 'mid-pregnancy' (days 170-240) and 'late pregnancy' (days 270-340), and the HRV values changed significantly in the second period only (Nagel et al., 2010, 2011). Although our possibilities were limited in terms of the number of mares available for the study, we could provide some basic data on HR and HRV in the fetuses of Lipizzaner mares which can support future studies in this field.

The mean FHR significantly decreased and the mean RR intervals (which were similar as in Nagel's study) tended to increase (in a non-significant degree in our study) during the ongoing pregnancy, in harmony with the findings of Nagel et al. $(2010,2011)$.

However, the standard deviation of FHR (SDFHR) significantly decreased in relation to gestational age in our study. These data showed that HRV in the foal fetus decreased as the pregnancy progressed, which is in contrast with the results of earlier equine studies (Nagel et al., 2010). The SDNN value in our study decreased from the 5th-7th month of gestation to term, but increased slightly in the 11th month (Table 1). It is important to note that we obtained higher values $(70 \pm 25$ and $166 \pm 108 \mathrm{msec})$ than the previous studies (Nagel et al., 2010, 2011). The RMSSD values decreased during the equine pregnancy (from $105 \pm 69$ to $77 \pm 37 \mathrm{msec}$ ), but this decrease was not significant. As HRV has been reported to decrease in horses under certain conditions such as stress, 
pain and fetal distress (Bowen, 2010), it is an important question whether a decrease in FHRV occurs in the foal fetus or there is a physiological decrease in FHRV during maturation. It has been described by van Leeuwen et al. (2013) that HRV is influenced by HR and a higher HR is generally associated with a lower HRV. In horses, fetal HR in months 5-11 of gestation is approx. between 115 and $80 \mathrm{bpm}$; therefore, the decreasing HRV observed during pregnancy cannot be explained by this fact. In human medicine, there have been detailed studies on the fetal maturation process and its effect on HR and HRV, and HRV parameters are also available for human fetuses. However, in the case of pregnant mares and their fetuses this is a relatively new field of perinatology and only limited information is available for researchers.

In the case of the four mare-fetus pairs that we could examine monthly towards the end of the pregnancy, we could see that the plots of the mean HR of the fetuses (Fig. 3) showed some differences. Although we had only four fetuses that could be compared, it is an interesting question whether there is an individual variation in HR between equine fetuses. In humans, the existence of high- and lowHR fetuses has been demonstrated (van Leeuwen et al., 2013), but in horses this has never been investigated so far. If there existed an intra-individual effect on HR and HRV of the foal fetus, we would have to consider the available data when examining a pregnant mare to determine whether her fetus is a high- or a low-HR fetus.

Using telemetric ECG, we could detect the fetal signal of the heart on the 121st day of pregnancy for the first time, earlier than others (Nagel et al., 2010, 2011) in previous studies. Therefore we plan to examine pregnant mares from the fourth and fifth months of gestation in the next breeding season.

In summary, we conclude that the analysis of fetal heart rate and heart rate variability is a promising tool for the assessment of fetal well-being, but further investigations on a higher number of animals are needed to evaluate its applicability in everyday clinical and stud practice.

\section{Acknowledgements}

We would like to thank the Szent István University Research Fund for financial support, Andor Dallos, Tamás Mikó and their colleagues at the Hungarian National Stud for technical help and Klaus Engel for continuous technical support during the study.

\section{References}

Adamson, S. L. (1999): Arterial pressure, vascular input impedance, and resistance as determinants of pulsatile blood flow in the umbilical artery. Eur. J. Obstet. Gynecol. Reprod. Biol. 84, 119-125.

Baska-Vincze, B., Baska, F. and Szenci, O. (2014): Evaluation of fetal well-being by transabdominal ultrasonography in the mare. Preliminary results [in Hungarian, with English abstract]. Magyar Állatorvosok Lapja 136, 195-204. 
Baska-Vincze, B., Rózsás, J., Baska, F. and Szenci, O. (2013): Veterinary evaluation of fetal wellbeing in the horse: Literature review [in Hungarian, with English abstract]. Magyar Állatorvosok Lapja 135, 323-331.

Bowen, I. M. (2010): Chapter 10 - Ambulatory electrocardiography and heart rate variability. In: Marr, C. M. and Bowen, I. M. (eds) Cardiology of the Horse. Second Edition. W. B. Saunders, Edinburgh. pp. 127-137.

Bucca, S., Fogarty, U., Collins, A. and Small, V. (2005): Assessment of feto-placental well-being in the mare from mid-gestation to term: Transrectal and transabdominal ultrasonographic features. Theriogenology 64, 542-557.

Cordero, M., Brorsen, B. W. and McFarlane, N. (2012): Circadian and circannual rhythms of cortisol, ACTH, and melanocyte-stimulating hormone in healthy horses. Domest. Anim. Endocrin. 43, 317-324.

Kovács, L., Nagy, K., Szelényi, Z., Szenci, O. and Tőzsér, J. (2012): Heart rate variability as a measurement of stress in cattle: biological background, methods and results. Literature review [in Hungarian, with English abstract]. Magyar Állatorvosok Lapja 134, 515-523.

Kovács, L., Tőzsér, J., Bakony, M. and Jurkovich, V. (2013): Changes in heart rate variability of dairy cows during conventional milking with nonvoluntary exit. J. Dairy Sci. 96, 7743-7747.

Long, W. A. and Henry, G. W. (1998): Autonomic and central neuroregulation of fetal cardiovascular function. In: Polin, R. A. and Fox, W. W. (eds) Fetal and Neonatal Physiology. W. B. Saunders, Philadelphia. pp. 943-961.

Minero, M., Canali, E., Ferrante, V. and Carenzi, C. (2001): Measurement and time domain analysis of heart rate variability in dairy cattle. Vet. Rec. 149, 772-774.

Mohr, E., Langbein, J. and Nürnberg, G. (2002): Heart rate variability: A noninvasive approach to measure stress in calves and cows. Physiol. Behav. 75, 251-259.

Nagel, C., Aurich, J. and Aurich, C. (2010): Determination of heart rate and heart rate variability in the equine fetus by fetomaternal electrocardiography. Theriogenology 73, 973-983.

Nagel, C., Aurich, J. and Aurich, C. (2011): Heart rate and heart rate variability in the pregnant mare and its foetus. Reprod. Domest. Anim. 46, 990-993.

Nörr, J. (1921): Fötale Elektrokardiogramme vom Pferd. Z. Biol. 73, 123-128.

Palmer, J. (2000): Fetal monitoring. Proceedings of the Equine Symposium and Annual Conference, San Antonio, TX, 2000. pp. 39-43.

R Core Team (2013): R: A Language and Environment for Statistical Computing. R Foundation for Statistical Computing, Vienna, Austria. URL http://www.R-project.org/

Reef, V. B., Vaala, W. E., Worth, L. T., Spencer, P. A. and Hammett, B. (1995): Ultrasonographic evaluation of the fetus and intrauterine environment in healthy mares during late gestation. Vet. Radiol. Ultrasound 36, 533-541.

Reef, V. B., Vaala, W. E., Worth, L. T., Sertich, P. L. and Spencer, P. A. (1996): Ultrasonographic assessment of fetal well-being during late gestation: development of an equine biophysical profile. Equine Vet. J. 28, 200-208.

Senger, P. L. (2012): Pathways to Pregnancy and Parturition. 3rd edition. Current conceptions, Inc., Redmond. 381 pp.

Van Leeuwen, P., Lange, S., Bettermann, H., Grönemeyer, D. and Hatzmann, W. (1999): Fetal heart rate variability and complexity in the course of pregnancy. Early Hum. Dev. 54, 259-269.

Van Leeuwen, P., Cysarz, D., Edelhäuser, F. and Grönemeyer, D. (2013): Heart rate variability in the individual fetus. Auton. Neurosci.: Basic and Clinical 178, 24-28.

von Borell, E., Langbein, J., Després, G., Hansen, S., Leterrier, C., Marchant-Forde, J., MarchantForde, R., Minero, M., Mohr, E., Prunier, A., Valance, D. and Veissier, I. (2007): Heart rate variability as a measure of autonomic regulation of cardiac activity for assessing stress and welfare in farm animals - A review. Physiol. Behav. 92, 293-316.

Wheeler, T., Cooke, E. and Murrills, A. (1979): Computer analysis of fetal heart rate variation during normal pregnancy. Br. J. Obstet. Gynaecol. 86, 186-197. 